\title{
Étude du potentiel de l'Industrie 4.0 quant à la transformation de la PME manufacturière québécoise : Une analyse littéraire et expérimentale
}

\author{
Study of the potential of Industry 4.0 for the transformation of the \\ Quebec manufacturing SME: A literary and experimental analysis
}

\author{
Sébastien Gamache ${ }^{1}$, Georges Abdulnour ${ }^{2}$, Chantal Baril ${ }^{3}$ \\ ${ }^{1}$ Département de génie industriel, Université du Québec à Trois-Rivières, Québec, Canada, \\ sebastien.gamache@uqtr.ca \\ ${ }^{2}$ Département de génie industriel, Université du Québec à Trois-Rivières, Québec, Canada, \\ georges.abdulnour@uqtr.ca \\ ${ }^{3}$ Département de génie industriel, Université du Québec à Trois-Rivières, Québec, Canada, chantal.baril@uqtr.ca
}

RÉSUMÉ. Le besoin des entreprises de se démarquer sur le marché mondial devient de plus en plus important avec le manque croissant de main d'œuvre et la croissance de la concurrence accentuée par l'arrivée des technologies numériques dans l'environnement industriel, logistique et commercial. Les entreprises de toute taille tendent vers l'Industrie 4.0. Les PME manufacturières québécoises semblent toutefois en retard quant à la transformation numérique de leur organisation et de leurs processus. Ce projet de recherche a pour objectif de présenter l'état des PME manufacturières québécoises au niveau du numérique et d'identifier une méthode pertinente et les outils les plus appropriés pour encourager les PME manufacturières québécoises à évoluer efficacement vers un environnement 4.0. Une analyse de la revue de littérature et d'expériences en terrain québécois montrent que malgré le manque de ressources, la flexibilité, l'agilité et la proximité avec les clients représentent les caractéristiques principales des $\mathrm{PME}$ manufacturières québécoises. L'utilisation d'outils qui accélèrent la prise de décisions efficace et qui améliorent la relation avec les clients semblent donc à privilégier dans ce type d'environnement.

ABSTRACT. The need for companies to differentiate themselves in the global market is becoming increasingly important with the growing lack of manpower and the growth of competition accentuated by the arrival of digital technologies in the industrial, logistic and commercial environment. Companies of all sizes are moving towards Industry 4.0. Quebec manufacturing SMEs, however, seem to lag behind in the digital transformation of their organization and processes. This research project aims to present the state of Quebec manufacturing SMEs at the digital level and to identify a relevant method and the most appropriate tools to encourage Quebec manufacturing SMEs to move efficiently towards a 4.0 environment. An analysis of the review of literature and experiences in Quebec shows that despite the lack of resources, the flexibility, the agility and the proximity to the customers are the main characteristics of Quebec manufacturing SMEs. The use of tools that accelerate effective decision-making and improve the relationship with customers therefore seem to be favoured in this type of environment.

MOTS-CLÉS. Industrie 4.0, transformation numérique, PME, PME manufacturière.

KEYWORDS. Industry 4.0, digital transformation, SME, manufacturing SME.

\section{Introduction}

Avec la chute des barrières internationales et l'arrivée des technologies numériques dans l'environnement manufacturier et commercial, les entreprises de toutes tailles doivent faire preuve d'ingéniosité pour demeurer concurrentielles. Pour se distinguer, les entreprises doivent miser sur la valeur ajoutée pour le client. Les technologies numériques et l'utilisation des données massives ont amené aux entreprises une nouvelle manière de générer de la valeur.

En 2011, à la foire annuelle d'Hanovre, l'Institut des Fraunhofer dévoilait une nouvelle politique gouvernementale allemande pour améliorer les pratiques industrielles à la grandeur du pays : 
l'Industrie 4.0. L'Industrie 4.0 est définie par Blanchet (2016) comme une politique industrielle développée par le gouvernement allemand ayant pour objectif de gagner et maintenir un avantage compétitif mondial au niveau des entreprises manufacturières. De par l'investissement dans les nouvelles technologies (infonuagique, automatisation, systèmes cyber-physiques, etc.), l'Industrie 4.0 se voit une solution d'avenir pour les industries manufacturières. Ces dernières doivent en effet répondre à un changement des consommateurs qui les force à aller vers la production de masse personnalisée. Elles doivent donc changer leurs paradigmes et leurs pratiques d'affaires. Pour ces raisons, l'Industrie 4.0 est de plus en plus utilisée dans les grandes industries allemandes. D'autres pays comme la Chine, la France et les États-Unis mettent des efforts importants dans la numérisation de leur industrie dans l'objectif de gagner un avantage compétitif mondial. Pour survivre à cette compétition, les entreprises québécoises doivent alors adhérer à la philosophie du 4.0 ainsi qu'à la transformation industrielle qui l'accompagne.

Ce projet de recherche tente d'évaluer l'état actuel des entreprises québécoises et d'identifier les moyens les plus significatifs, soulevés dans la littérature, pour faciliter la mise en œuvre de la transformation numérique des PME manufacturières (PMEM). Dans les pages suivantes, l'Industrie 4.0, la PME manufacturière, la méthode d'implantation du 4.0 et la mesure de performance choisie dans ce travail seront présentés, abordant les notions de valeur, de capacités organisationnelles et opérationnelles, de technologies 4.0, de compétences numériques et de transformation numérique. La Figure 1 montre les différents sujets abordés dans cet article.

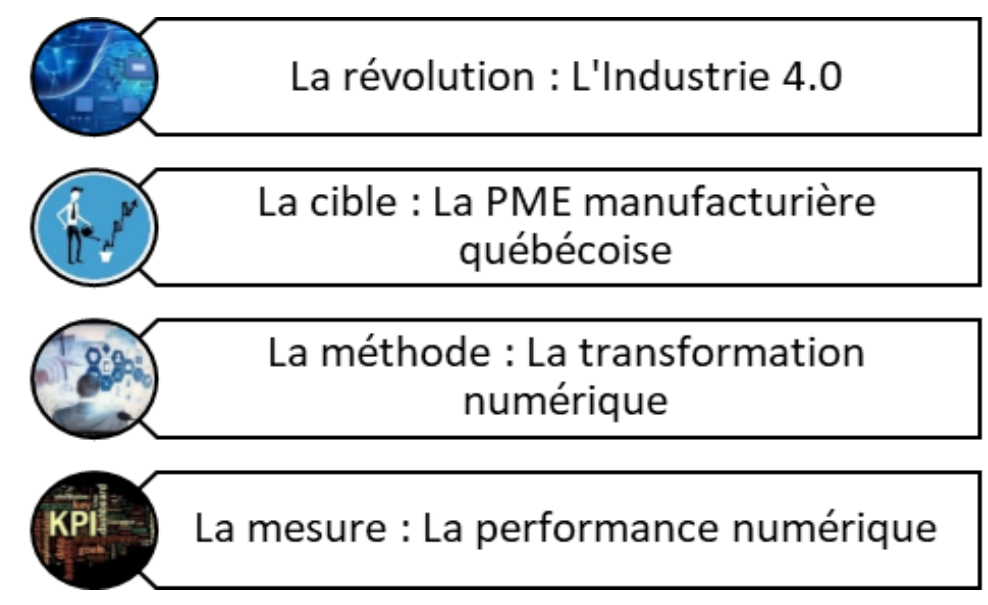

Figure 1. La révolution, la cible, la méthode et la mesure

La première section de l'article couvre la problématique, les questions de recherche ainsi que le but de l'étude. La stratégie adoptée pour la recension des écrits est abordée par la suite. La troisième partie présente la revue de littérature et son analyse. La quatrième section est dédiée à une comparaison entre la littérature et l'industrie québécoise. Enfin, une conclusion présente et synthétise les informations les plus marquantes du travail exécuté.

\section{Problématique de recherche et objectifs}

Dans tous les secteurs d'activités industrielles, académiques et commerciales, les technologies numériques occupent une place croissante et sont en continuel développement. Les technologies de l'information et des communications (TIC) ont déjà fait leurs preuves au niveau des gains qu'elles peuvent apporter à une organisation. En conséquence, elles sont de plus en plus intégrées aux pratiques d'affaires. Dans un contexte de mondialisation et d'omniprésence du numérique, les petites et moyennes entreprises manufacturières deviennent vulnérables aux géants de l'industrie, ainsi qu'aux start-ups, qui peuvent rapidement prendre le contrôle du marché. Les exigences de la 
personnalisation de masse et la concurrence mondiale amènent les industries québécoises à devoir offrir une nouvelle valeur, soit par l'amélioration de leur offre, de leur efficacité et de leur agilité. Pour y arriver, plusieurs outils existent, dont les technologies numériques, par exemple, qui permettent de générer de la valeur à plusieurs niveaux.

Les grandes industries allemandes telles que Siemens, Bosch et Volkswagen occupent des positions de leaders dans l'implantation du numérique, principalement véhiculées par l'Industrie 4.0. En effet, elles intègrent et investissent de plus en plus dans les technologies numériques, la connectivité, la robotisation et l'intelligence des systèmes (Kohler et Weisz, 2016).

Au Québec, on n'entend que depuis peu parler de l'Industrie 4.0 et on remarque peu d'adhésion concrète à cette-dite révolution. En raison d'un manque de ressources, notamment au niveau des connaissances techniques et théoriques ou du financement approprié, peu d'efforts concrets sont mis en rapport à leur transformation numérique. Comment les PME québécoises peuvent-elles évoluer vers un environnement « 4.0 »? Pour répondre à cette question, il est nécessaire de définir ce qui est entendu par le terme «Industrie 4.0 ». Il est également pertinent de déterminer une méthode pour mesurer la capacité d'évolution d'une entreprise dans ce type d'environnement. Le terme utilisé dans cet article pour représenter cette capacité est la performance numérique. Cet indicateur est utilisé afin de situer les PME manufacturières sur une échelle de progression « 4.0 » et cibler les actions à poser par les entreprises et autres parties prenantes telles que le gouvernement pour améliorer la capacité d'évolution des entreprises québécoises dans l'environnement 4.0 et les aider à atteindre leurs objectifs d'affaires. L'objectif principal de cet article est alors de présenter l'état des PME manufacturières québécoises au niveau du numérique et identifier les solutions numériques les plus pertinentes dans leur contexte afin de les aider à évoluer efficacement dans un environnement 4.0.

\section{Stratégie de la revue de littérature}

Une exploration de la littérature a été effectuée afin de faire le point sur l'état des connaissances actuelles concernant l'Industrie 4.0 et les principaux outils numériques et leurs impacts sur la petite et moyenne entreprise manufacturière. Les moteurs de recherche SCOPUS, ScienceDirect, Springer et Google Scholar ont permis de cibler différents documents tels que des livres, articles scientifiques, rapports de recherche, sites internet, conférences et vidéos portant sur l'Industrie 4.0, la numérisation, l'agilité, l'économie, l'automatisation, le management et le leadership pour couvrir le sujet dans sa globalité. Des ouvrages datant des années 1970 jusqu'à 2018 ont permis d'observer la chronologie et l'évolution de l'économie globale et des industries dans le temps. La structure de la recherche présentée à la Figure 2 a permis de guider et d'organiser les idées principales issues de la revue de la littérature. Plus spécifiquement, la revue de la littérature est divisée en trois parties, soit l'Industrie 4.0, la PME manufacturière et la transformation numérique. 


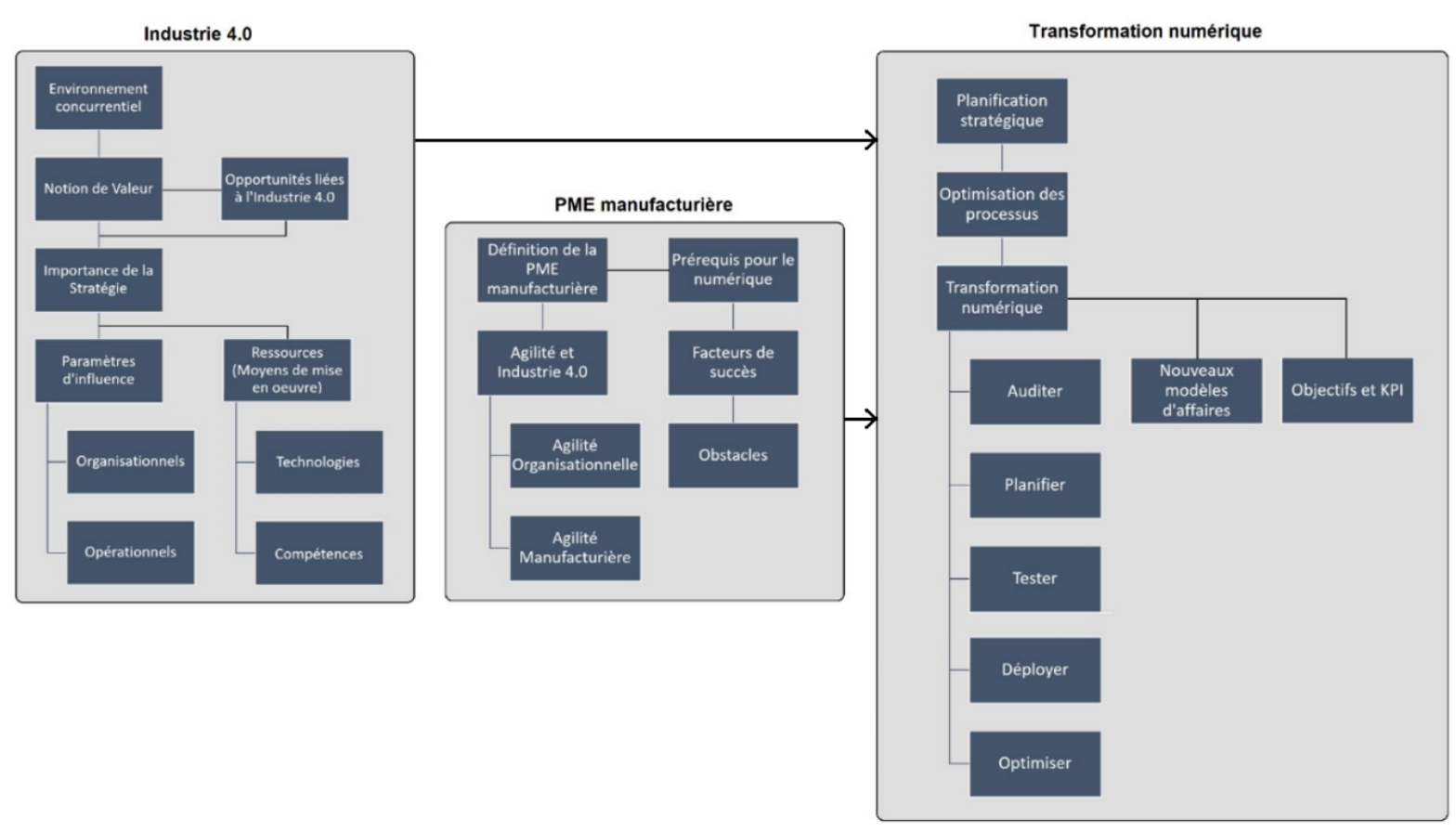

Figure 2. Structure de la recherche

\section{Revue de la littérature}

\section{Partie 1. La révolution : industrie 4.0}

Tel qu'il est démontré dans les prochaines pages, l'Industrie 4.0 n'est pas une mode ou une tendance à proprement parler. Il provient d'une chaîne d'événements qui ont amené à chercher de nouvelles solutions pour demeurer compétitif, répondre à l'environnement inconstant, incertain, complexe et ambigüe et pallier au manque de main d'œuvre vécu dans la plupart des entreprises manufacturières (Blanchet, 2016). Au Canada et au Québec par exemple, le taux de chômage moyen se situait respectivement à $6 \%$ et $5,5 \%$ en août 2018 , occasionnant régulièrement le refus de contrats, des retards de livraison et un niveau élevé d'heures supplémentaires (ISQ, 2018). Cette évolution de l'environnement concurrentiel, vécue dans plusieurs pays, entre autres, par l'accessibilité et la prise de conscience du potentiel des technologies a poussé au développement de l'Industrie 4.0 (Blanchet, 2016).

\section{Environnement concurrentiel et valeur}

Michael Porter (1979) a développé le modèle des 5 forces pour l'analyse de sa position concurrentielle. L'auteur démontre la structure concurrentielle des entreprises en fonction des cinq axes stratégiques suivants :

- Le pouvoir de négociation des clients ;

- Le pouvoir de négociation des fournisseurs ;

- La menace des produits ou services de substitution ;

- La menace d'entrants potentiels sur le marché ;

-L'intensité de la rivalité entre les concurrents.

Selon l'auteur, une entreprise doit étudier son environnement en considérant ces 5 caractéristiques pour limiter les menaces potentielles et développer son avantage concurrentiel. Avec l'arrivée massive des technologies numériques dans le milieu manufacturier, les clients ont accès à plus de choix et d'informations, les fournisseurs ont accès à un plus grand bassin de clients, 
les produits sont de plus en plus substitués par des alternatives numériques et mobiles $(\mathrm{CD}$, films, consoles de jeux, applications...), les startups ont accès à plus de ressources, grâce notamment au crowdfunding, crowdsourcing, et incubateurs, et la rivalité devient de plus en plus forte à l'intérieur et à l'extérieur d'un même secteur d'activité (Weinman, 2015).

Le modèle de Porter se concentre spécifiquement sur l'impact de l'environnement d'une entreprise dans le développement de son avantage concurrentiel. L'article de J. Barney (1991) intitulé «Firm Resources and Sustained Competitive Advantage » fût l'un des premiers ouvrages à traiter de la valeur basée sur les ressources (Resource-Based Value-RBV). Contrairement à Porter, Barney présente les moyens internes que possède une entreprise pour générer un avantage concurrentiel. Ce cadre de gestion se base sur le fait que toutes les entreprises possèdent un mix de ressources hétérogènes, qui ont un potentiel de génération de valeur unique. Dans la théorie de la $\mathrm{RBV}$, la valeur, ou d'avantage compétitif, provient de l'utilisation de ressources qui doivent respecter quatre critères :

- Les ressources doivent posséder une valeur - comme répondre à un besoin, réduire les coûts, augmenter les revenus, éliminer une menace, etc. ;

- Les ressources doivent être rares

-Les ressources doivent être difficiles à imiter

-Les ressources ne doivent pas être facilement remplaçables

Selon Barney, une entreprise doit alors évaluer la façon qu'elle utilise ses ressources et développer la stratégie appropriée pour répondre à ces critères et se différencier de la concurrence. Le CEFRIO (2016), Avasant (2016) et Weinman (2015) démontrent que dans un environnement hautement numérique, le facteur de différenciation devient encore plus important et que la valeur peut se développer en misant sur le produit et le service; le processus; ou la relation-client. Ces auteurs affirment d'ailleurs que les nouvelles technologies, inhérentes à l'Industrie 4.0, permettent désormais de collecter et analyser des données et revoir ses modèles d'affaires, ses produits et services, ses processus et améliorer l'implication et la relation avec les clients.

Kohler et Weisz (2016) démontrent que l'intégration des nouvelles technologies, encouragé par l'Industrie 4.0, crée un environnement fortement compétitif. Pour demeurer compétitives, les entreprises doivent donc trouver une manière de se distinguer et développer un avantage concurrentiel via, notamment les nouveaux potentiels de génération de valeur.

\section{Le potentiel de l'Industrie 4.0}

Hermann et al. (2015) ont mené une revue de 51 publications et ont fait ressortir les termes et concepts les plus fréquemment associés à l'Industrie 4.0. Les auteurs affirment alors que les principes clés reliés à l'Industrie 4.0, ou à la numérisation des industries, sont l'interopérabilité, la virtualisation, la décentralisation, la capabilité en temps réel, l'orientation service et la modularité, possibles grâce à l'implantation de ces différentes technologies. Il faut rappeler toutefois que la plupart des articles portant sur l'Industrie 4.0 ont été rédigés en considérant la grande entreprise et non la PME. Par exemple, comme Mœuf (2018) le présente, les systèmes cyber-physiques sont peu utilisés dans les petites et moyennes entreprises en raison du coût en infrastructure élevé.

Porter et Heppelmann (2014) démontrent que les nouvelles technologies numériques, intelligentes et connectées, sont la clé pour avancer vers un environnement 4.0. Les auteurs définissent l'intelligence des produits par leur capacité à collecter et analyser des données. La connectivité est, quant à elle, la capacité à transférer les données d'un objet à un autre pour faciliter le transfert d'information, la prise de décision, et l'analyse plus avancée des données collectées. Les auteurs 
présentent la forte relation entre les besoins de gestion d'une entreprise et le niveau de numérisation requis. Les différents niveaux de numérisation sont, selon Porter et Heppelmann (2014) : La surveillance (monitoring); le contrôle; l'optimisation; et l'automatisation. En fonction de ce qui est recherché par une solution numérique, il est possible que seul un niveau de surveillance ne soit requis. Dans une autre application, une solution numérique pourra complètement automatiser une tâche. L'établissement d'une stratégie d'affaires claire devient alors un prérequis, voire la première étape, pour entamer le passage à l'Industrie 4.0 (CEFRIO, 2016).

\section{Stratégie d'affaires}

Largement appuyé par la littérature, Miles et Snow (1978) présentent trois types de stratégie d'affaires principales, soit la stratégie prospective, analytique ou défensive. L'idée étant reprise par Raymond (2009), ce dernier affirme qu'une entreprise est de type prospectif lorsque sa stratégie consiste à introduire des nouveaux produits et services, revoir ses modèles d'affaires et constamment rechercher des nouveaux marchés (Miles et Snow, 1978). Ce type d'entreprise doit miser sur la flexibilité et l'agilité de ses systèmes pour absorber les changements et maintenir un niveau de rentabilité satisfaisant. Une entreprise de type analytique préfère aller vers des produits ou des services déjà éprouvés, mais en optant pour l'optimisation de ses processus pour offrir des produits de meilleure qualité à meilleurs coûts (Raymond, 2009). Elle doit alors développer une infrastructure efficace et flexible pour offrir une meilleure efficience et rentabilité que les prospecteurs et pour répondre rapidement aux changements de l'environnement (Miles et Snow, 1978). Une entreprise du type défensif est davantage orientée coût, contrôle de ses processus et conservation de sa position sur le marché (Raymond, 2009). Elle se concentre dans une niche pointue et tente de développer une place stable par des coûts compétitifs et une qualité inégalée, mais évite d'aller dans des marchés non-maîtrisés (Miles et Snow, 1978). Au regard de cette typologie, et bien que la stratégie définisse la direction d'une entreprise, la mise en œuvre doit passer par l'acquisition, le développement et le déploiement d'outils et de techniques organisationnels et opérationnels, de compétences humaines et d'une infrastructure technologique qui facilite l'atteinte de ses objectifs d'affaires.

\section{Paramètres d'influence de la performance numérique}

Dans la littérature, plusieurs éléments et moyens de mise en œuvre sont soulevés comme méthode de déploiement de la transformation numérique. Le choix de ces méthodes dépend notamment de l'orientation stratégique, des enjeux, du contexte interne et externe et des ressources disponibles, tant humaines que technologiques dans les entreprises. Pour orienter les priorités, les investissements et les vecteurs de valeur dans une entreprise de plus en plus numérique, plusieurs auteurs ont développé des modèles théoriques qui ont pour objectif de faciliter la prise de décision et l'atteinte des objectifs d'affaires via différents outils et techniques liés à l'Industrie 4.0. Les idées principales recueillies sont présentées au Tableau 1. 


\begin{tabular}{|c|c|c|c|c|c|c|c|c|c|c|c|c|c|c|c|c|c|c|c|}
\hline $\begin{array}{l}\text { Paramètres d'influence de la performance } \\
\text { numérique }\end{array}$ & 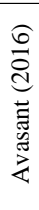 & 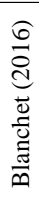 & 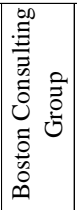 & 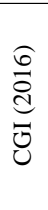 & 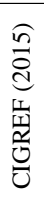 & 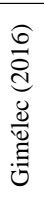 & 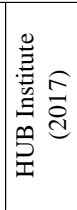 & 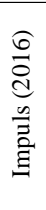 & 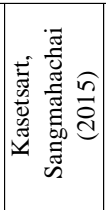 & 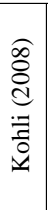 & 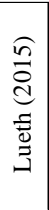 & 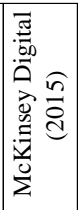 & 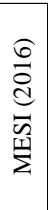 & 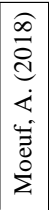 & 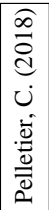 & 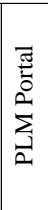 & 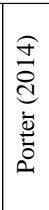 & 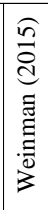 & 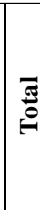 \\
\hline Acquisition et développement des compétences & $\mathrm{x}$ & $\mathrm{x}$ & $\mathrm{x}$ & & $\mathrm{x}$ & & $\mathrm{x}$ & $\mathrm{x}$ & & $\mathrm{x}$ & & & $\mathrm{x}$ & & & $\mathrm{x}$ & & & 9 \\
\hline Cybersécurité & & & $\mathrm{x}$ & $\mathrm{x}$ & & $\mathrm{x}$ & $\mathrm{x}$ & $\mathrm{x}$ & $\mathrm{x}$ & & & & & $\mathrm{x}$ & & $\mathrm{x}$ & $\mathrm{x}$ & & 9 \\
\hline Vision et stratégie & $\mathrm{x}$ & & $\mathrm{x}$ & $\mathrm{x}$ & $\mathrm{x}$ & & $\mathrm{x}$ & $\mathrm{x}$ & & $\mathrm{x}$ & & & & & $\mathrm{x}$ & $\mathrm{x}$ & & & 9 \\
\hline Agilité et innovation & $\mathrm{x}$ & & $\mathrm{x}$ & & $\mathrm{x}$ & & $\mathrm{x}$ & $\mathrm{x}$ & & & & & $\mathrm{x}$ & & & $\mathrm{x}$ & & $\mathrm{x}$ & 8 \\
\hline Automatisation des processus & & $\mathrm{x}$ & $\mathrm{x}$ & $\mathrm{x}$ & $\mathrm{x}$ & & & $\mathrm{x}$ & & & $\mathrm{x}$ & & $\mathrm{x}$ & & & $\mathrm{x}$ & & & 8 \\
\hline Maîtrise des technologies & $\mathrm{x}$ & $\mathrm{x}$ & $\mathrm{x}$ & $\mathrm{x}$ & $\mathrm{x}$ & & $\mathrm{x}$ & & & & & & & & & $\mathrm{x}$ & $\mathrm{x}$ & & 8 \\
\hline Utilisation opérationnelle des données & $\mathrm{x}$ & & $\mathrm{x}$ & $\mathrm{x}$ & $\mathrm{x}$ & & $\mathrm{x}$ & $\mathrm{x}$ & & & & $\mathrm{x}$ & & & $\mathrm{x}$ & & & & 8 \\
\hline Utilisation stratégique des données & $\mathrm{x}$ & & $\mathrm{x}$ & $\mathrm{x}$ & $\mathrm{x}$ & & $\mathrm{x}$ & $\mathrm{x}$ & & & & $\mathrm{x}$ & & & $\mathrm{x}$ & & & & 8 \\
\hline Ouverture à l'externe et collaboration & $\mathrm{x}$ & & & $\mathrm{x}$ & $\mathrm{x}$ & $\mathrm{x}$ & $\mathrm{x}$ & & & & & & & $\mathrm{x}$ & $\mathrm{x}$ & & & & 7 \\
\hline e-Commerce et marketing numérique & $\mathrm{x}$ & & $\mathrm{x}$ & & & $\mathrm{x}$ & $\mathrm{x}$ & & & & & & $\mathrm{x}$ & & & $\mathrm{x}$ & & & 6 \\
\hline Organisation du travail (Lean et AC) & & $\mathrm{x}$ & $\mathrm{x}$ & & $\mathrm{x}$ & $\mathrm{x}$ & & & & & & & & & $\mathrm{x}$ & $\mathrm{x}$ & & & 6 \\
\hline Orientation service & $\mathrm{x}$ & & $\mathrm{x}$ & & & & $\mathrm{x}$ & $\mathrm{x}$ & & & & & & $\mathrm{x}$ & & $\mathrm{x}$ & & & 6 \\
\hline Personnalisation & & & $\mathrm{x}$ & & & $\mathrm{x}$ & $\mathrm{x}$ & & $\mathrm{x}$ & & $\mathrm{x}$ & & & $\mathrm{x}$ & & & & & 6 \\
\hline Cocréation et innovation ouverte & & & & & $\mathrm{x}$ & $\mathrm{x}$ & $\mathrm{x}$ & & & & & $\mathrm{x}$ & & & & & & $\mathrm{x}$ & 5 \\
\hline Investissement et ressources disponibles & & & $\mathrm{x}$ & $\mathrm{x}$ & $\mathrm{x}$ & & & $\mathrm{x}$ & & $\mathrm{x}$ & & & & & & & & & 5 \\
\hline Nouveaux modèles d'affaires & & & $\mathrm{x}$ & $\mathrm{x}$ & & & $\mathrm{x}$ & & & & & & & & $\mathrm{x}$ & $\mathrm{x}$ & & & 5 \\
\hline Écosystème et architecture numérique & & & $\mathrm{x}$ & & & & $\mathrm{x}$ & $\mathrm{x}$ & & & & & & & & $\mathrm{x}$ & & & 4 \\
\hline Communication interne & & & & & $\mathrm{x}$ & & $\mathrm{x}$ & $\mathrm{x}$ & & & & & & & & & & & 3 \\
\hline Engagement et fidélisation & $\mathrm{x}$ & & & & $\mathrm{x}$ & & $\mathrm{x}$ & & & & & & & & & & & & 3 \\
\hline Gestion des données (collecte, qualité, livraison) & & & & & & & & $\mathrm{x}$ & & & & $\mathrm{x}$ & & & & $\mathrm{x}$ & & & 3 \\
\hline Engagement et exemplarité & & & & & & & $\mathrm{x}$ & & & & & & & & & & & & 1 \\
\hline Gestion du changement & & & & & & & $\mathrm{x}$ & & & & & & & & & & & & 1 \\
\hline Veille technologique & & & & $\mathrm{x}$ & & & & & & & & & & & & & & & 1 \\
\hline
\end{tabular}

Tableau 1. Paramètres d'influence de la performance numérique

Le Tableau 1 montre que certains éléments tels que l'acquisition et le développement des compétences, la cybersécurité, la définition de sa vision et stratégie, l'agilité et l'innovation, l'intégration, la connexion et la maîtrise des technologies et l'utilisation des données sont au cœur de l'Industrie 4.0, car ils apparaissent très souvent dans la littérature. D'autres éléments comme la gestion du changement, l'engagement et l'exemplarité de la direction et la veille technologique sont moins abordés, mais montrent tout de même un intérêt pour la transition au numérique. Deux modèles ressortent du Tableau 1 par leur pertinence. Il s'agit du modèle d'IMPULS et celui du HUB $\underline{\text { Institute. }}$

La Fondation de la Fédération de l'Ingénierie Allemande IMPULS a conduit en 2016 une étude en collaboration avec plusieurs experts tels que l'Institut de Cologne sur la Recherche Économique et l'Institut du Management Industriel de l'Université Aachen sur le readiness numérique des entreprises allemandes. L'étude présente 6 volets d'évaluation, à savoir: La stratégie et l'organisation; l'usine intelligente; les opérations intelligentes; les produits intelligents; les services par l'analyse des données; et les employés. Le modèle d'IMPULS soulève de nombreux points d'intérêts à considérer dans l'évaluation des moyens de mise en œuvre à développer dans une entreprise. Des plus pertinents, on peut soulever : L'infrastructure des équipements; l'utilisation des données; la sécurité des TI; l'autonomie des processus; le partage d'information; l'analyse des données en temps réel; les services offerts par l'analyse des données; l'acquisition et le développement des compétences des employés; la stratégie numérique de l'entreprise; et la gestion de l'innovation.

Le modèle du HUB Institute (Ducrey et Vivier, 2017) a également pour but d'aider les entreprises à exécuter leur transformation numérique. Selon les auteurs, six dimensions principales doivent être adressées relativement à la stratégie d'affaires, notamment : Le leadership; la culture et l'organisation; la gestion des technologies; la gestion des données; le système de mesure (processus décisionnel); et l'expérience client. Le leadership détermine la direction de l'entreprise, sa connaissance du marché et de l'évolution de l'environnement. La culture et l'organisation 
représentent les moyens mis en œuvre pour favoriser l'innovation, l'amélioration continue, l'acceptation du changement, l'acquisition de compétences, etc. La gestion des technologies aborde la maturité numérique, la cybersécurité, et l'utilisation de technologies numériques pour l'atteinte de ses objectifs d'affaires. La gestion des données représente la captation, l'entreposage, le traitement et la qualité des données utilisées dans l'entreprise. Le système de mesure représente la façon d'utiliser les données pour faciliter le processus décisionnel. Enfin, l'expérience client représente les méthodes utilisées pour personnaliser et améliorer l'offre et les relations avec le client.

\section{Moyens de mise en cuvre du 4.0}

La concrétisation d'une stratégie passe d'abord par le déploiement de ressources, tant humaines que financières, technologiques et matérielles. Barney (2001) a défini les ressources d'une entreprise comme étant «tous les actifs, les capacités, les processus organisationnels, les attributs, les informations et les connaissances contrôlés par une entreprise, qui permettent de concevoir et implanter des stratégies qui améliorent son efficacité et son efficience », voire créer de la valeur sur le marché. Autrement dit, les ressources d'une entreprise représentent l'ensemble des moyens, tant humains que matériels, qui permettent la mise en œuvre de la stratégie d'affaires. Cette sous-section présente en ce sens les notions de ressources humaines (compétences, connaissances, habiletés, attitudes) et les différentes technologies actuellement disponibles pour les PME, qui facilitent la mise en œuvre de sa stratégie organisationnelle.

\section{Ressources humaines}

Le CEFRIO (2016) affirme que la capacité d'une organisation repose à la fois sur l'usage de ses actifs en place mais aussi, des compétences de ses employés. En ce sens, Caldeira et Ward (2003) défendent que les compétences sont composées de connaissances, d'habiletés et d'attitudes qui favorisent l'exécution d'une tâche. L'individu possède différentes habiletés techniques, connaissances et savoir-être qui lui sont propres. Dans un environnement hautement numérique, les compétences numériques prennent davantage d'importance. Le CEFRIO (2016) définit les compétences numériques comme l'habileté des individus à utiliser les technologies numériques pour :

- Analyser, sélectionner et évaluer de manière critique l'information numérique ;

- Résoudre des problèmes ;

-Développer une base de connaissances collaboratives tout en s'engageant dans les pratiques organisationnelles.

Dans ce même rapport, les auteurs affirment que les compétences doivent être développées sur trois plans, à savoir le plan technique, collaboratif et cognitif. Les compétences techniques représentent 1'habileté des individus à utiliser efficacement les technologies. Les compétences collaboratives sont la capacité à collaborer avec les technologies, utiliser les technologies pour établir des canaux de communication efficaces et résoudre différents problèmes. Enfin, les compétences cognitives représentent la capacité à traiter et analyser des informations en tenant compte de la pertinence et la fiabilité de l'information traitée.

Considérant l'avancement rapide des technologies, le développement des compétences des individus devient de plus en plus critiques pour les organisations. Il devient alors important de bien comprendre ce que sont les différents outils technologiques du 4.0 et ce qu'ils peuvent apporter dans une entreprise. 


\section{Outils technologiques du 4.0}

Avec l'omniprésence de l'Internet et le développement rapide de toutes sortes de technologies, les entreprises doivent identifier et sélectionner celles qui sont les plus pertinentes pour leur réalité. Comme le mentionne le CEFRIO (2017), l'investissement dans des technologies pertinentes mène à une exécution efficace de sa stratégie d'affaires. La revue de littérature de nombreux auteurs a permis de faire ressortir les groupes de technologies numériques reliées à l'Industrie 4.0.

Blanchet (2016) propose une catégorisation de ces différentes technologies via 5 contextes d'utilisation, à savoir : La conception de produit et processus; le pilotage et contrôle; les opérations de fabrication; les services; et l'organisation du travail.

La conception des produits et des processus inclue l'utilisation de logiciels de simulation, de conception et fabrication assistées par ordinateur, de gestion du cycle de vie du produit, etc. Le pilotage et le contrôle représente l'utilisation de CPS, d'Internet des Objets, de logiciels de traçabilité et de gestion de flux tels que des véhicules guidés automatiquement, des outils de GPAO, de gestion de chaîne d'approvisionnement et l'utilisation de RFID. Les opérations de fabrication incluent les machines intelligences et autonomes, les cobots et la fabrication additive pour améliorer la précision, l'efficacité et la flexibilité. Les services incluent tout ce qui est maintenance prédictive, Big Data, Analytique, télémaintenance et système de gestion de la clientèle (CRM). Enfin, l'organisation du travail inclue la réalité virtuelle et augmentée, ainsi que des pratiques «nontechnologiques » telles que le lean manufacturing et le Juste-À-Temps et l'acquisition et le développement des compétences numériques. Le Tableau 2 montre l'ensemble des moyens technologiques disponibles pour la mise en œuvre de la transformation numérique d'une entreprise en fonction des différents auteurs, regroupés à l'aide de la catégorisation de Blanchet (2016). Il est à noter que certaines technologies se retrouvent dans plus d'une catégorie et ont été dupliquées pour respecter le regroupement de Blanchet.

\begin{tabular}{|c|c|c|c|c|c|c|c|c|c|c|c|c|c|c|c|c|c|c|}
\hline & Outils technologiques du 4.0 & 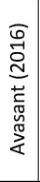 & 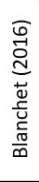 & 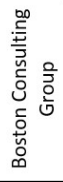 & 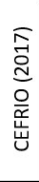 & $\begin{array}{l}\bar{o} \\
\stackrel{-}{0} \\
\underline{D} \\
\bar{U}\end{array}$ & 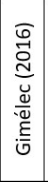 & 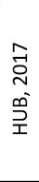 & $\begin{array}{l}\bar{\Xi} \\
0 \\
d \\
\underline{\omega} \\
\overline{\bar{a}} \\
\underline{\underline{\Xi}}\end{array}$ & 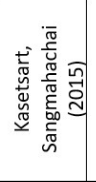 & 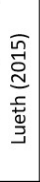 & 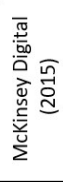 & 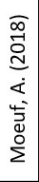 & 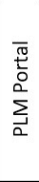 & 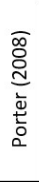 & 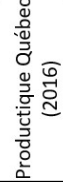 & 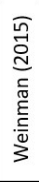 & 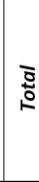 \\
\hline \multirow{2}{*}{$\begin{array}{l}\text { Conception } \\
\text { des produits } \\
\text { et processus }\end{array}$} & Fabrication additive & & $\mathrm{x}$ & $\mathrm{x}$ & & & $\mathrm{x}$ & & & $\mathrm{x}$ & $\mathrm{x}$ & $x$ & & $\mathrm{x}$ & & $\mathrm{x}$ & & 8 \\
\hline & Simulation & & & $\mathrm{x}$ & $\mathrm{x}$ & & $\mathrm{x}$ & & $\mathrm{x}$ & & & $\mathrm{x}$ & $x$ & & & & & 6 \\
\hline \multirow{6}{*}{$\begin{array}{l}\text { Pilotage et } \\
\text { contrôle }\end{array}$} & Big Data, Analytics et BI & $x$ & $x$ & $x$ & $x$ & $x$ & $\mathrm{x}$ & $x$ & $\mathrm{x}$ & $x$ & $\mathrm{x}$ & & $\mathrm{x}$ & $\mathrm{x}$ & $\mathrm{x}$ & $x$ & $x$ & 15 \\
\hline & Internet des Objets & $\mathrm{x}$ & $x$ & $\mathrm{x}$ & $\mathrm{x}$ & $\mathrm{x}$ & $x$ & $\mathrm{x}$ & & $\mathrm{x}$ & $x$ & $\mathrm{x}$ & $\mathrm{x}$ & $x$ & & $\mathrm{x}$ & $\mathrm{x}$ & 14 \\
\hline & Infonuagique & $x$ & & $\mathrm{x}$ & $\mathrm{x}$ & $x$ & $\mathrm{x}$ & $x$ & $\mathrm{x}$ & $\mathrm{x}$ & $\mathrm{x}$ & & $x$ & $\mathrm{x}$ & $x$ & & $x$ & 13 \\
\hline & Chaîne d'approvisionnement intelligente & & & $\mathrm{x}$ & $\mathrm{x}$ & & $\mathrm{x}$ & & & $\mathrm{x}$ & $\mathrm{x}$ & $\mathrm{x}$ & & $\mathrm{x}$ & & & & 7 \\
\hline & Systèmes Cyber-physiques & $\mathrm{x}$ & & & $\mathrm{x}$ & & & $\mathrm{x}$ & & $\mathrm{x}$ & $x$ & & $x$ & & & & & 6 \\
\hline & $\begin{array}{l}\text { Applications et logiciels (WMS, MES, ERP, Traçabilité, } \\
\text { Planification) }\end{array}$ & & & & $\mathrm{x}$ & & & $\mathrm{x}$ & & & & & & & $x$ & $\mathrm{x}$ & & 4 \\
\hline \multirow{2}{*}{$\begin{array}{l}\text { Opérations } \\
\text { de } \\
\text { fabrication }\end{array}$} & Communication M2M & & & & $\mathrm{x}$ & & $\mathrm{x}$ & & & & & & $x$ & $x$ & & & & 4 \\
\hline & Intelligence artificielle & & & & $\mathrm{x}$ & & & $\mathrm{x}$ & & & & & & & & & & 2 \\
\hline \multirow{7}{*}{ Services } & Big Data, Analytics et BI & $x$ & $x$ & $\mathrm{x}$ & $x$ & $x$ & $\mathrm{x}$ & $x$ & $\mathrm{x}$ & $x$ & $\mathrm{x}$ & & $x$ & $\mathrm{x}$ & $\mathrm{x}$ & $x$ & $\mathrm{x}$ & 15 \\
\hline & Cybersécurité & & & $x$ & $\mathrm{x}$ & $x$ & $\mathrm{x}$ & & $x$ & $\mathrm{x}$ & $\mathrm{x}$ & & $x$ & $x$ & $x$ & $\mathrm{x}$ & & 11 \\
\hline & Maintenance prédictive & & $\mathrm{x}$ & $\mathrm{x}$ & $\mathrm{x}$ & & $\mathrm{x}$ & & & & $\mathrm{x}$ & $\mathrm{x}$ & & & & $\mathrm{x}$ & & 7 \\
\hline & Mobilité & $\mathrm{x}$ & & & & & & & & & $x$ & & & $x$ & & $x$ & $\mathrm{x}$ & 5 \\
\hline & Applications et logiciels (CRM, télémaintenance...) & & & & $\mathrm{x}$ & & & $\mathrm{x}$ & & & & & & & $\mathrm{x}$ & $\mathrm{x}$ & & 4 \\
\hline & Médias sociaux & $\mathrm{x}$ & & & $\mathrm{x}$ & & & $\mathrm{x}$ & & & & & & & & & $\mathrm{x}$ & 4 \\
\hline & Internet des Services, commerce électronique & $\mathrm{x}$ & & & $\mathrm{x}$ & & & & & $\mathrm{x}$ & & & & & & & & 3 \\
\hline $\begin{array}{c}\text { Organisation } \\
\text { du travail }\end{array}$ & Réalité augmentée / virtuelle & & $\mathrm{x}$ & $\mathrm{x}$ & $\mathrm{x}$ & & $\mathrm{x}$ & $\mathrm{x}$ & & & $\mathrm{x}$ & $\mathrm{x}$ & $\mathrm{x}$ & & & & & 8 \\
\hline
\end{tabular}

Tableau 2. Différents outils technologiques du 4.0

Le Tableau 2 présente les différents outils technologiques selon leur niveau d'intérêt chez les auteurs. Certaines technologiques comme le Big Data, la Cybersécurité, l'Internet des Objets et 
l'infonuagique sont mentionnés dans plus de $60 \%$ des cas, démontrant le caractère important de la donnée dans l'Industrie 4.0. Certaines autres technologies telles que les différents systèmes informatiques (ERP, MES, WMS, traçabilité, planification, CRM...), les véhicules guidés automatiquement, la mobilité, la communication M2M, l'intelligence artificielle, les médias sociaux et l'Internet des Services sont, quant à elles, abordées dans moins de $25 \%$ des cas, possiblement pour des raisons de non-pertinence ou de manque de connaissances et d'expertise. Il est également possible que certaines technologies, comme les logiciels informatiques, soient davantage associées à la troisième révolution industrielle plutôt qu'à la quatrième, expliquant leur maigre présence dans la littérature associée au 4.0.

La catégorisation de Blanchet (2016) permet de rassembler l'ensemble des outils technologiques associés à l'Industrie 4.0. Elle permet également de déterminer le lieu d'impact d'une technologie et oriente, en ce sens, le choix d'une solution pour une entreprise dépendamment de son besoin. On note par ailleurs que lorsqu'ils abordent l'Industrie 4.0, la plupart des auteurs mentionnent les outils de pilotage et de contrôle ainsi que des nouveaux services offerts aux clients. Pour beaucoup, l'automatisation et les systèmes de production sont la base de l'Industrie 4.0. Ce constat n'est toutefois pas remarqué lorsqu'on aborde les outils technologiques concrets tels que la production assistée par les robots, l'utilisation de robots collaboratifs, la communication M2M et l'intelligence artificielle. Ce constat soulève alors la question de la dominance de l'impact du pilotage et du contrôle ainsi que du développement de nouveaux services par rapport à celui des opérations de fabrication.

Comme il a été présenté dans cette partie, il existe de nombreux éléments qui affectent les multiples définitions de l'Industrie 4.0 dans la littérature, notamment la grosseur des entreprises qui désirent implanter le 4.0. Considérant son origine, ses objectifs variés, les nombreux moyens de mises en œuvre, l'impact sur l'emploi et l'utilisation des différents outils, il est néanmoins possible de simplifier l'Industrie 4.0 par l'utilisation des technologies numériques et des données en temps réel pour améliorer la prise de décision, l'efficacité et l'agilité dans les processus administratifs et opérationnels, tout en permettant la personnalisation de masse, tant au niveau des produits et des services offerts. Les ressources des petites et moyennes entreprises sont limitées et que bien que les moyens de mise en œuvre présentés dans la présente section aient tous un intérêt, les PME manufacturières doivent cibler les différents outils et techniques à développer en fonction de leur importance respective, de leur environnement manufacturier, de leurs enjeux, leurs objectifs, leur clientèle visée et la concurrence existante. Il convient en ce sens de présenter la PME et ses particularités, enjeux et les ressources dont elles disposent pour se démarquer sur le marché.

\section{Partie 2. La cible : La PME manufacturière}

Sommer (2015) affirme que l'Industrie 4.0 n'encourage que les grandes entreprises. Selon l'auteur, cette philosophie pourra avoir comme conséquence d'augmenter le fossé entre les PME et les grandes industries. En effet, les PME ont généralement moins de ressources et de connaissances vis-à-vis les dernières tendances technologiques (Bendis, 2004). Le manque de numérisation des procédés et la faible performance numérique des petites et moyennes entreprises pourrait éventuellement affecter les PME par rapport à leurs concurrents internationaux du type Amazon et Google. Sommer (2015) affirme que les PME ne sont pas encore prêtes à faire le saut de la $4{ }^{\text {ème }}$ révolution industrielle. Un sondage auprès de 2000 entreprises démontre que les PME manquent d'informations par rapport à cette nouvelle révolution. De plus, elles ne se sentent souvent pas soutenues par le gouvernement, ne savent pas comment et par où commencer et n'ont pas confiance dans la sécurité et la protection des données. Il existe néanmoins de nombreux avantages issus de l'Industrie 4.0 qui peuvent être adaptés dans la PME manufacturière. Il convient alors de définir ce qu'est une PME et les caractéristiques qui lui sont propres. 
Le concept des PME varie dépendamment de l'auteur qui traite du sujet ainsi que du secteur géographique. En ce sens, plusieurs critères, autant quantitatifs que qualitatifs, peuvent être utilisés pour décrire une PME.

Selon l'OCDE, la PME possède entre 10 et 249 employés et génère moins de 50 millions d'euros annuellement. Au Canada, une PME est considérée comme une entreprise dont le nombre d'employés est inférieur à 500 et que le chiffre d'affaires ne dépasse pas 50M\$. Malgré la valeur frontière entre la moyenne et la grande entreprise qui varie entre l'Europe et le Canada, on remarque que plus de 97\% des PME au Québec emploient moins de 100 employés (Statistique Canada, 2015). Dans le secteur de fabrication au Canada en 2015, 19602 entreprises sur 50902 (38,5\%) employaient entre 10 et 99 employés. Les microentreprises (1 et 4 employés) et très petites entreprises (5 et 9 employés) étaient dénombrées à 17768 (34,9\%) et 10119 (19,9\%) respectivement dans le même rapport. Les entreprises employant entre 100 et 499 employés étaient du nombre de $3121(6,1 \%)$ et celles avec plus de 500 employés de 292 (0,6\%). Dans l'Enquête sur la population active; et calculs d'Innovation, Sciences et Développement économique Canada publiée par Statistique Canada, on constate que 49,4\% des emplois dans le domaine de la fabrication se trouvent dans les entreprises de 1 à 99 employés.

Hormis ces critères quantitatifs, Bendis (2004) a décrit la PME comme une entreprise avec des ressources humaines, matérielles et financières limitées. Néanmoins, leur flexibilité et leur proximité avec les clients, et se matérialisent via une structure organisationnelle souple, la vitesse de réponse à l'environnement, la qualité du service à la clientèle et le style de gestion aplati (Man et al., 2002). Un comparatif des forces et faiblesses de PME est présenté au Tableau 3.

\begin{tabular}{|l|l|}
\hline Faiblesses & Forces \\
\hline Manque de ressources financières (Bendis, 2004) & $\begin{array}{l}\text { Flexibilité organisationnelle et opérationnelle (Man et al., } \\
\text { 2002) }\end{array}$ \\
\hline Difficulté à recruter de la main-d'œuvre (Bendis, 2004) & Proximité avec les clients (Man et al., 2002) \\
\hline Souvent éloignée des grands centres & Accès à du financement gouvernemental (Bendis, 2004) \\
\hline Pas ou peu d'accès aux économies d'échelle & Structure organisationnelle souple (Man et al., 2002) \\
\hline $\begin{array}{l}\text { Manque de connaissances techniques et académiques (nouvelles } \\
\text { technologies, nouveaux modes de gestion, etc.) (Mœuf, 2018) }\end{array}$ & Vitesse de réponse à l'environnement (Man et al., 2002) \\
\hline $\begin{array}{l}\text { Centralisation du pouvoir et des décisions (Mœuf, 2018) } \\
\text { Manque de stratégie à long terme (Mœuf, 2018) }\end{array}$ & Engagement et impact de la direction (Mœuf, 2018) \\
\hline & Créativité et innovation très présentes (Man et al., 2002) \\
\hline
\end{tabular}

Tableau 3. Caractéristiques principales des PME

Ces informations illustrent bien les caractéristiques des PME manufacturières québécoises tant au niveau des avantages que des inconvénients qui leur sont associés. Pour résumer, les PME sont considérées ici comme des entreprises embauchant entre 10 et 99 employés, possédant des connaissances et ressources qui sont limitées et qui doivent faire appel à l'innovation, le travail d'équipe ainsi que l'agilité manufacturière et organisationnelle pour répondre au marché et faire face à la concurrence mondiale. En ce sens, l'agilité organisationnelle et opérationnelle devient pertinente pour le marché québécois qui tente de répondre à l'adaptation des entreprises dans un environnement dynamique, imprévisible et turbulent (Nagel et Dove, 1991). La prochaine section traite alors de ces notions comme solutions potentielles à la réalité des PME manufacturières.

Agilité

Dans un environnement hautement numérique et pour développer un avantage concurrentiel, les PME doivent continuellement innover tant au niveau de leurs produits et services que de leurs 
processus (CEFRIO, 2016). Comme l'a démontré le CIGREF (2015), l'agilité dans l'organisation, mais aussi au niveau des opérations, devient nécessaire aux entreprises pour générer de la valeur et se démarquer de la concurrence.

Le CIFREG (2015) définit l'agilité comme un synonyme d'adresse et de vivacité. Plus exactement, l'agilité est souvent présentée comme «la capacité d'une entreprise à croître dans un environnement marqué par un changement continu et imprévisible d'un marché global, caractérisé par une demande de qualité supérieure, de haute performance, de faible coût et de produits et services correspondant aux exigences des consommateurs » (Nagel et Dove, 1991; Goldman, 1994). Le construit d'agilité organisationnelle a évolué au cours des années vers de nombreuses définitions, mais un consensus de quatre capacités, ou composantes, de l'agilité organisationnelle a eu tendance à revenir entre les années 1990 et 2000. Ces capacités sont la réactivité, la rapidité, la flexibilité et les compétences.

Réactivité - ou la réponse à un changement : Sharifi et al. (2001) définissent la réactivité comme la capacité à identifier et anticiper les changements de l'environnement et d'y répondre de manière réactive et proactive.

Rapidité - ou la vitesse de prise de décision: La rapidité issue de l'agilité organisationnelle est définie comme le délai pour prendre une décision et la mettre en œuvre (Eisenhardt, 1989).

Flexibilité - ou la capacité de s'adapter aux changements : Huang et Cullen (2001) définissent la flexibilité organisationnelle comme la manière de s'adapter aux variations de la demande interne et externe. Concrètement, il s'agit de se procurer des ressources (main d'œuvre, équipements, logiciels...) polyvalentes et adaptables aux besoins changeants de l'organisation.

Compétences - ou les capacités humaines face au changement : Selon Prahalad et Hamel (1990), le terme «compétence » représente principalement l'apprentissage collectif dans une organisation. De son côté, Ferrante (2006) définit les compétences comme un ensemble évolutif de capacités qui permettent d'atteindre des objectifs organisationnels de façon efficace et efficiente. Enfin, pour reprendre la définition employée précédemment dans cet article, les compétences peuvent aussi être définies comme l'ensemble des connaissances, habiletés et attitudes des individus qui favorisent l'exécution d'une tâche (Caldeira et Ward, 2003).

En bref, 1'Industrie 4.0 prend ses racines du besoin des entreprises à gagner en efficacité et en flexibilité pour répondre aux exigences de la personnalisation de masse. L'agilité représente alors la base qui permet aux entreprises de survivre dans l'environnement issu de l'Industrie 4.0. Dans un contexte où la main d'œuvre spécialisée se fait plus rare, l'agilité, alliée à l'utilisation des technologies du 4.0, permet de prendre des décisions plus rapidement et plus efficacement tout en s'équipant de machines, de systèmes et d'employés polyvalents qui s'adaptent facilement et rapidement à la demande des clients.

\section{Partie 3. La méthode : La transformation numérique}

La transformation numérique représente la démarche encouragée par certains auteurs concernant l'intégration des technologies numériques dans ses pratiques d'affaires (HUB Institute, 2017). Selon certains auteurs, la planification stratégique avec des objectifs clairs, le contrôle de ses processus grâce au lean manufacturing et une connaissance du numérique sont des prérequis à la transformation numérique (CEFRIO, 2016; HUB Institute, 2017; Little, A.D., 2017). La Banque de Développement du Canada (2018) a défini la planification stratégique comme l'exercice de mise en place d'une saine stratégie d'affaires qui permet de guider ses décisions et ses actions dans un but de croissance à long terme. 
Tel que présenté par le CIGREF (2015), la personnalisation de masse, proposée par l'Industrie 4.0, passe par une flexibilité opérationnelle et un délai de réaction rapide, autrement dit, par l'agilité opérationnelle. Comme l'ont démontré Abdulnour et al. (1999), l'agilité manufacturière passe par les outils du lean manufacturing le contrôle total de la qualité, l'aménagement cellulaire, la maintenance, la réduction des temps de mise en course, l'élimination des gaspillages, l'amélioration continue, les Kaizen, les Kanbans, et les petits lots de production. Les principes de réduction des gaspillages et de l'amélioration continue deviennent donc nécessaires pour les entreprises désirant aller dans la direction du 4.0.

Le HUB Institute (2017) propose une approche en cinq étapes qui permet de faciliter le succès de la transformation numérique. Les étapes sont les suivantes :

-Auditer : L'audit représente selon les auteurs, l'étape de prise de connaissance de son état actuel permettant de mesurer l'état d'une entreprise en fonction de ses capacités organisationnelles et opérationnelles et de la maîtrise de ses processus afin de faire ressortir les forces et les opportunités d'amélioration et d'établir les priorités en fonction de ses enjeux et de ses objectifs d'affaires.

-Planifier : L'étape de la planification évoque le développement d'un plan détaillé des différents projets d'amélioration, priorisés en fonction de leur niveau d'urgence, de la disponibilité des ressources matérielles, humaines, financières et temporelles et de la préséance respective des projets.

- Tester : Au lieu de mettre en place des projets d'envergure de longue durée, le HUB Institute se base sur l'agilité et les petits projets rapidement viables. Cette méthode de tests permet de voir rapidement les failles d'un projet ainsi que les gains apportés et permet d'apporter les corrections dès le départ plutôt qu'à la toute fin du projet.

- Déployer : Le déploiement représente l'implantation des projets à l'ensemble de l'organisation une fois le projet validé à l'étape précédente. Le HUB Institute y inclus les tâches et actions concrètes à accomplir, les moyens humains et financiers, les objectifs, les délais et les responsabilités de chaque intervenant.

-Optimiser : L’optimisation revient à dire « contrôle ». Cette dernière étape est en effet basée sur l'amélioration continue, l'apport d'ajustements et la création de nouvelles priorités. L'optimisation inclus la mesure de la performance, le contrôle des situations problématiques et le retour à l'étape d'audit.

\section{État des PME manufacturières québécoises au niveau du numérique}

À la suite d'une étude des résultats de 148 cartographies de chaînes de valeur effectuées dans des PME québécoises, Gamache et al. (2017) ont démontré que la plupart des entreprises manufacturières de petite taille n'avait pas totalement adhéré à l'ère de l'Industrie 4.0. En effet, selon les résultats de l'étude, seulement près du tiers des entreprises cartographiées employaient des outils informatiques tels que les ERP ou MES pour collecter, traiter et transmettre des données de qualité et faciliter la prise de décision. L'étude soulève également que l'absence de données pertinentes à une gestion en temps réel et le manque d'outils de planification de la production, d'aménagement d'usine adéquat, de systèmes d'assurance qualité et d'intégration du Juste-À-Temps démontrent le retard des entreprises quant à leur numérisation. Le projet de cartographie avait, dans la totalité des cas, comme intérêt de mettre en exergue les opportunités d'amélioration des processus des entreprises pour gagner en efficacité, flexibilité et agilité, démontrant l'intention des entrepreneurs à demeurer compétitif. On remarque toutefois un manque de ressources pour le faire. 
Dans la même lignée, un rapport du CEFRIO publié en 2017 traite des outils de l'Industrie 4.0 qui sont les plus présents et maitrisés dans les industries québécoises. Ce dernier montre que les suites bureautiques, les sites web informationnels, les logiciels de dessins, les progiciels de gestion et les médias sociaux sont les outils technologiques les plus largement utilisés avec des pourcentages respectifs de $71 \%, 53 \%, 46 \%, 34 \%$ et $30 \%$ sur un échantillon de 312 entreprises. Les systèmes de production flexibles (FMS), les systèmes de gestion du cycle de vie des produits (PLM), l'intelligence d'affaires (BI), les systèmes de gestion d'entrepôt (WMS), les robots, les systèmes d'exécution de la production (MES), le Web Analytics, les sites web transactionnels et les systèmes de gestion de la relation client (CRM) sont, quant à eux, utilisés dans moins de $18 \%$ des cas. Le CEFRIO explique que l'accès limité à l'Internet haute vitesse en région et la maturité numérique limitée des entreprises posent un frein au passage au numérique. Les entreprises qui ont intégrés quelques outils numériques ont par ailleurs mentionné dans $54 \%$ des cas avoir ressenti des effets positifs significatifs sur leurs activités quotidiennes au niveau de la conception et de la fabrication des produits.

Une étude terrain dans 15 entreprises, effectuée en 2017 dans le cadre d'un mandat avec le Ministère de l'Économie, de la Science et de l'Innovation, a montré que la performance numérique moyenne des entreprises étudiées était de 65\%, avec un écart-type de 9,7\%. Les activités de veille technologique, l'utilisation de systèmes de planification à capacité finie, l'utilisation d'instructions de travail à jour et le pilotage d'usine en temps réel ont démontré avoir un effet significatif positif sur la performance numérique des entreprises. L'étude montre également que l'infonuagique, la planification de la production via un système ERP, l'utilisation de données en temps réel, la présence d'un plan numérique et l'emploi des pratiques du lean tendent à augmenter la performance des entreprises. Dans cette même étude, et en reprenant les dimensions de la performance numérique du HUB Institute (2017), les plus grandes forces des entreprises sont les activités liées au niveau du leadership, de la culture et de l'organisation. Les plus grandes opportunités d'amélioration sont, quant à elles, au niveau des opérations de fabrication et du pilotage et contrôle.

En France, Mœuf et al. (2017) ont démontré que la plupart des technologies du 4.0 employée dans les entreprises étaient surtout celles avec un coût d'acquisition plus faible telles que l'Internet des Objets, l'Infonuagique et les systèmes de simulation. Les plus gros systèmes tels que les outils du Big Data, les Systèmes Cyber-Physiques, les robots collaboratifs, le M2M et la réalité virtuelle sont jugés trop dispendieux en rapport au retour sur investissement et requièrent trop de changements internes. Une telle étude n'a pas été trouvée dans la littérature quant aux industries québécoises, mais les constats peuvent néanmoins être posés comme hypothèse dans ce contexte. Une étude similaire pourra être intéressante dans une future recherche.

En comparaison avec la littérature, il est possible de voir que les PME manufacturières québécoises vivent des défis similaires aux autres PME, notamment au niveau du manque de connaissances, de ressources et de vision. Le faible score issu des opérations de fabrication, du pilotage et du contrôle dans la performance numérique des PME québécoises ainsi que le peu d'effort entrepris dans les activités lean démontre que la transformation numérique ne fait que débuter. La littérature montre clairement que le numérique comporte un potentiel de développement important pour les PME, mais cette sensibilisation ne semble pas avoir totalement atteint le terrain des PME manufacturières. Le développement de stratégies et l'apport de connaissances au niveau des paramètres d'influence du 4.0 et des outils existants devraient apporter de l'aide aux PME pour poursuivre, voire entamer, leur transformation numérique. 


\section{Conclusion}

Tel que présenté en début d'article, le but de cette recherche est de présenter l'état des PME manufacturières québécoises au niveau du numérique et d'identifier une méthode pertinente et les outils les plus appropriés pour encourager les PME manufacturières québécoises à évoluer efficacement vers un environnement 4.0. La revue de littérature ainsi que son analyse ont permis une meilleure compréhension des enjeux vécus par les PME manufacturières, mais aussi la démystification de l'Industrie 4.0 et le potentiel issu de la transformation numérique des PMEM. La démarche proposée ainsi que le regroupement des outils numériques permettront d'orienter les actions à entreprendre et les outils à acquérir en fonction du besoin réel d'une entreprise.

La structure de la revue de la littérature présentée a permis de proposer un lien clair entre les différentes notions de l'Industrie 4.0, de la PME manufacturière et de la transformation numérique. Ceci a permis d'organiser les concepts et les modèles théoriques de sorte à offrir une compréhension ordonnée de l'interaction entre ces différents éléments. Cette structure pourra être utilisée dans de futurs travaux pour expliquer la provenance et l'impact de l'Industrie 4.0.

Les échantillons de 148, 312 et 15 entreprises des expériences terrain montrent des résultats similaires au niveau des conclusions, notamment au niveau du besoin en performance numérique, mais aussi en performance organisationnelle en général. L'homogénéité des études permet également de comparer l'état de l'industrie québécoise avec d'autres pays tels que la France, mais tout en ciblant les forces et opportunités d'amélioration spécifiques à l'industrie québécoise. Comme en France toutefois, malgré le nombre croissant d'outils et technologies numériques, la plupart n'est pas encore implantée dans les PMEM québécoises.

L'Industrie 4.0 a été définie dans cette recherche comme l'utilisation des technologies numériques et des données en temps réel pour améliorer la prise de décision, l'efficacité et l'agilité dans les processus administratifs et opérationnels. Les auteurs ont démontré que les nombreux outils associés au 4.0 permettent en ce sens d'améliorer la conception des produits et processus, la fabrication, le pilotage et le contrôle, les nouveaux services et l'organisation du travail. Le besoin d'agilité, la proximité avec le client et l'importance de la donnée ont toutefois ressorti comme des caractéristiques primordiales dans la PME manufacturières québécoise qui désire aller vers le 4.0. Les technologies numériques 4.0 associées au pilotage et au contrôle ainsi qu'à la création de nouveaux services telles que l'infonuagique, l'utilisation intégrée de la donnée en temps réel (ex : intelligence d'affaires, tableaux de bord numériques, etc.), l'Internet des Objets, la maintenance prédictive et à distance, mais aussi les outils du lean manufacturing semblent alors offrir des opportunités plus adaptées aux PMEM québécoises.

On peut remarquer certaines limitations dans la recherche actuelle. En effet, des échantillons de 148,312 et 15 entreprises demeurent limitées considérant le nombre important d'entreprises différentes et diversifiées au Québec. Le type de produit fabriqué, le mode de production et le secteur d'activité sont toutes des variables qui peuvent affecter significativement le niveau de numérisation atteint et requis dans les entreprises. Les méthodes et les outils nécessaires à cette numérisation peuvent également varier d'une entreprise à l'autre. Les regroupements proposés sont généraux et n'incluent qu'une quinzaine d'articles. Considérant le nombre croissant d'articles rédigés sur l'Industrie 4.0 dans les dernières années, les paramètres d'influence et les outils technologiques peuvent démontrer des importances variables. Le choix des articles scientifiques étudiés est également aléatoire et issu d'une recherche étalée sur plusieurs mois. Il est possible que de nouveaux articles aient été publiés entre temps et qui ne soient pas considérés dans la recherche. Il sera intéressant pour des travaux futurs d'expérimenter la démarche de transformation numérique proposée par le HUB Institute avec pour mesure la performance numérique afin de cibler les outils avec le plus de potentiel pour les industries, mais aussi en considérant l'impact du secteur d'activité, 
le type de produit et le mode de production des entreprises. La mesure de l'impact des outils sur la performance numérique pourra aider les PME manufacturières à cibler de façon éclairée les outils avec le plus de potentiel pour leur réalité. Enfin, la mesure de la performance numérique sur le terrain permettra de voir les forces ainsi que les opportunités d'amélioration les plus importantes dans l'industrie québécoise.

\section{Références}

Abdul-Nour, G., Drolet, J. et Lambert, S. (1999) Mixed production, flexibility and SME. Computers \& Industrial Engineering 37 (1999) 429-432

Avasant (2016) Digital Enterprise Transformation: Rebooting Business Services for the New Global Economy. CreateSpace Independent Publishing Platform. ISBN 978-1514704066

Barney, J. B., "Firm Resources and Sustained Competitive Advantage." Journal of Management, Vol. 17, No. 1, 1991, pp. $99-120$

BCG The Boston Consulting Group (2015) Industry 4.0. The future of productivity and growth in manufacturing industries. Avril 2015. [En ligne], consulté le 4 juin 2018 <https://www.zvw.de/media.media.72e472fb-1698-4a158858-344351c8902f.original.pdf>

BDC, Planification stratégique, [En ligne], consulté le 4 juin 2018, <https://www.bdc.ca/fr/consultation/pages/planification-strategique.aspx >

Bendis, R.A. (2004) Technology-based economic development. Defense Related SME's : Analysis and description of current conditions. F.D. Carvalho (Ed.) IOS Press, 2004

Blanchet, M. (2016) Industrie 4.0, Nouvelle donne industrielle, nouveau modèle économique. Lignes de repères. ISBN 978-2-36609-043-7

Bloem J, Doorn M van, Duivestein S, Excoffier D, Maas R, Ommeren E va. The Fourth Industrial Revolution Things to Tighten the Link Between It and Ot,. 2014.

Caldeira, M. M. et Ward, J. M. (2003) Using resource-based theory to interpret the successful adoption and use of information systems and technology in manufacturing small and medium-sidez enterprises. European Journal of Information System. Volume 12, pp. 127-141

CEFRIO (2016) Prendre part à la révolution manufacturière? Du rattrapage technologique à l'industrie 4.0 chez les PME. Numérique et Entreprise. [En ligne] consulté le 11 mars 2017, <http://www.cefrio.qc.ca/publications/numerique-entreprise/industrie4-0-chez-les-pme-du-quebec/>

CEFRIO (2017) Portrait des pratiques numériques des entreprises manufacturières de la région de la capitalenationale. Québec International. ISBN : 978-2-923852-75-1

CGI (2016) Industrie 4.0. Pour une entreprise hautement concurrentielle. [En ligne], consulté le 4 juin 2018, <https://www.cgi.com/sites/default/files/white-papers/manufacturing_industry-4_white-paper-fr.pdf>

CIGREF (2015) L'agilité dans l'entreprise. Modèle de maturité. Octobre 2015. [En ligne], consulté le 4 juin 2018, $<$ http://www.cigref.fr/wp/wp-content/uploads/2015/11/CIGREF-2015-Agilite-dans-1-entreprise-Modele-deMaturite.pdf>

Ducrey, V. et Vivier, E. - HUB Institute (2017) Le guide de la transformation digitale. Eyrolles, Hub Management, pp. 328.

Eisenhardt, K. M. (1989). Making fast strategic decisions in high-velocity environments. Academy of Management Journal, 32(3): 543-576.Ferrante, G. (2006) 25 ans d'agilité organisationnelle : clarification et opérationnalisation du construit. Thèse de doctorat, Université Grenoble Alpes. Sciences de la gestion, 7 août 2006.

Gamache, S., Abdulnour, G. et Baril, C. (2016) Industrie 4.0 dans les PME québécoises : Bilan et premiers constats. 12th International Conference on Industrial Engineering

Gimélec (2014) Industrie 4.0. Les leviers de la transformation. [En ligne], consulté le 4 juin 2018 <http://www.gospi.fr/IMG/pdf/industrie_4.0_les_leviers_de_la_transformation2014-gimelec-min.pdf>

Goldman, S. L., 1994, Agile competition and virtual corporations, National Forum, 74 (2), 43-49. 
Hermann, M., Pentek, T. et Otto, B. (2015) Design Principles for Industrie 4.0 Scenarios, Business Engineering Institute St. Gallen, Lukasstr. 4, CH-9008 St. Gallen. accessed on 4 May 2016

Huang, H. et Cullen, J. B. (2001) Labour flexibility and related HRM practices : A study of large Taiwanese manufacturers. Revue Canadienne des sciences de l'administration, vol. 18, no. 1. P. 33-39

Impuls (2016) Industry 4.0 Readiness. [En ligne], consulté le 11 mars 2017, <https://www.industrie40readiness.de/?lang=en>

Industrie Canada (2012) "Recherche et statistique sur la PME", [En ligne], consulté le 06-12-2014 <https://www.ic.gc.ca/eic/site/061.nsf/fra/01278.html>

Institut de la Statistique du Québec (2018) Résultat de l'Enquête sur la population active du Québec [En ligne], consulté le 17 septembre 2018. <http://www.stat.gouv.qc.ca/statistiques/travail-remuneration/resultats-epa201808.pdf>

Kagermann H, Wahlster W, Helbig J. Recommendations for implementing the strategic initiative INDUSTRIE 4.0. 2013.Kohler, D. et Weisz, J.-D. (2016) Industrie 4.0 - Les défis de la transformation numérique du modèle industriel allemand. La documentation française, France, ISBN : 978-2-11-010210-2

Kohli, R. et Grover, V. (2008) Business value of IT : An essay on expanding research directions ton keep up with the Times. Journal of the Association for Information Systems. Volume 9, no. 1. Article 2. Pp. 23-29, Janvier 2008

Little, A. D. (2017) Digital Lean Management - Unlock potential and achieve next performance levels www.adl.com/digitallean

Lueth, K.L. (2015) Will the industrial internet disrupt the smart factory of the future. <https://iotanalytics.com/industrial-internet-disrupt-smart-factory/>

Man, T.W.Y., Lau, T. et Chan, K.F. (2002), The competitiveness of small and medium enterprises a conceptualization with focus on entrepreneurial competencies, Journal of Business Venturing 17, pp. 123-142.

McKinsey Digital (2015) Industry 4.0. How to navigate digitization of the manufacturing sector. McKinsey\&Compagny

Miles, R. E. and C. C. Snow, Organizational Strategy, Structure, and Process. New York: McGraw-Hill, 1978.

Ministère de l'Économie, de la Science et de l'Innovation (MESI) (2016) Plan d'action en économie numérique. Stratégie numérique du Québec. Gouvernement du Québec 2016. Bibliothèque et Archives nationales du Québec. ISBN : 978-2-550-75695-8 (PDF)

Mœuf (2018) The industrial management of SMEs in the era of Industry 4.. International Journal of Production Research. Volume 56. Numéro 3

Moeuf, A. (2018) Identification des risques, opportunités et facteurs critiques de succès de l'industrie 4.0 pour la performance industrielle des PME. Thèse de doctorat de l'Université Paris-Saclay, avril 2018.

Nagel, R. N., \& Dove, R. 1991. 21st Century Manufacturing Enterprise Strategy: An Industry-Led View, Vol. 1: 58.

Organisation de Coopération et de Développement Économiques (OCDE) (2004) Les statistiques sur les PME : Vers une mesure statistique plus systématique du comportement des PME. $2^{\text {ème }}$ conférence de l'OCDE des ministres en charges des petites et moyennes entreprises (PME). Istanbul, Turquie, juin 2004

Pelletier, C. (2018) Présentation sur l'industrie 4.0. Travaux fondés sur Baltzan et Welsh (2015) et Laudon et Laudon (2016). Chambre de commerces et d'industries de Trois-Rivières. Mars 2018.

PLM Portal (2015) - PLM as enabler for industry 4.0, [En ligne], consulté le 27 mars 2017, <http://www.plmportal.org/en/ntt-data-plm-as-enabler-for-industry-4-0.html>

Porter, M. E. (1979) How Competitive Forces Shape Strategy, Harvard Business Review, mars-avril 1979, p. 137-145

Porter, M. E. (2008) Competitive Advantage: Creating and Sustaining Superior Performance. Simon and Schuster, ISBN: 1416595848

Porter, M. E. et Heppelmann, J. L. (2014) How smart, connected products are transforming competition. Harvard Business Review, volume 65. Novembre 2014

Prahalad, C. K., \& Hamel, G. 1990. The Core Competence of the Corporation. Harvard Business Review, 68(3): 7991. 
Raymond, L. et Croteau, A.-M., (2009) Manufacturing strategy and business strategy in medium-sized enterprises: Performance effects of strategic alignment. IEEE Transactions on engineering management, Vol. 56. No. 2. Mai 2009.

Rüßmann M, Lorenz M, Gerbert P, Waldner M, Justus J, Engel P, et al. Industry 4.0 The Future of Productivity and Growth in Manufacturing Industries. 2015.

Sangmahachai, K. (2015) Revolution to Industry 4.0. Kasetsart Energy and Technology Management Center

Sharifi, H., Colquhoun, G., Barclay, I., \& Dann, Z. 2001. Agile manufacturing: a management and operational framework. Proceedings of the Institution of Mechanical Engineers -- Part B -- Engineering Manufacture (Professional Engineering Publishing), 215(6): 857-869.

Sommer, L. (2015) Industrial Revolution - Industry 4.0. Are German Manufacturing SMEs. Journal of Industrial Engineering and Management, Volume 8, Numéro 5, pp. 1512-1532

Statistique Canada, Registre des entreprises, décembre 2015. <https://www.ic.gc.ca/eic/site/061.nsf/fra/h_03018.html>

Weinman, F. (2015) Digital Disciplines: Attaining Market Leadership via the Cloud, Big Data, Social, Mobile, and the Internet of Things. Wiley. ISBN 978-1118995396 